\title{
MULTI-STAGE APPOINTMENT SCHEDULING FOR OUTPATIENT CHEMOTHERAPY UNIT: A CASE STUDY
}

\author{
Asma Bouras $^{1}$, Malek Masmoudi ${ }^{2, *}$, Nour El Houda SaAdani ${ }^{3}, Z_{\text {Ied Bahroun }}^{4}$ \\ and Mohamed Amine Abdeljaouad ${ }^{5,6}$
}

\begin{abstract}
This paper deals with a multi stage hybrid flow-shop problem (HFSP) that arises in a privately Chemotherapy clinic. It aims to optimize the makespan of the daily chemotherapy activity. Each patient must respect the cyclic nature of chemotherapy treatment plans made by his referent oncologist while taking into account the high variability in resource requirements (treatment time, nurse time, pharmacy time). The problem requires the assignment of chemotherapy patients to oncologists, pharmacists, chemotherapy beds or chairs and nurses over a 1-day period. We provided a Mixed Integer Program (MIP) to model this issue, which can be considered as a five-stage hybrid flow-shop scheduling problem with additional resources, dedicated machines, and no-wait constraints. Since this problem is known to be NP-hard, we provided a lower bound expression and developed an approximated solving algorithm: a tabu search inspired metaheuristic based on a constructive heuristic that can quickly reach satisfying results. To assess the empirical performance of the proposed approach, we conducted experiments on randomly generated instances based on real-world data of a Tunisian private clinic: Clinique Ennasr. Computational experiments show the efficiency of the proposed procedures: The mathematical model provided optimal solutions in reasonable computational time only for small instances (up to 10 patients). Meta-heuristic's results demonstrate, also, that the proposed approach offers good results in terms of solution quality and computational times with an average relative gap to the MIP solution equal to $3.13 \%$ and to the lower bound equal to $5.37 \%$ for small instances (up to 15 patients). The same gap to the lower bound increases to $25 \%$ for medium and large size instances (20-50 patients).
\end{abstract}

Mathematics Subject Classification. 90C05 and 68W25.

Received August 8, 2018. Accepted February 20, 2021.

\footnotetext{
Keywords. Mathematical programming, heuristic, tabu search, patient flow, chemotherapy, scheduling, flow-shop, multiple resources.

1 Mediatron Lab, Higher School of Communications of Tunis, University of Carthage, Faculty of Sciences of Tunis, University of Tunis El Manar, Tunis, Tunisia.

2 Université de Lyon, Université Jean Monnet Saint-Etienne, Faculté des Sciences et Techniques, 42000 Saint Étienne, France.

3 Université de Tunis El Manar, F.S.T., LIP2-LR99ES18, 2092 Tunis, Tunisia.

4 Industrial Engineering, ESM Graduate Program, College of Engineering, American University of Sharjah, PO Box 26666, Sharjah, UAE.

5 CEA Tech Hauts-de-France, 59000 Lille, France.

6 LISPEN, Arts et Métiers Lille, 8, boulevard Louis XIV, 59046 Lille Cedex, France.

* Corresponding author: malek.masmoudi@univ-st-etienne.fr
} 


\section{INTRODUCTION}

Recently, the private healthcare sector has been in a continual development [30]. Indeed, despite the fact that private health care services are rather expensive, an increasing number of people tend to favour them over the public sector thanks to their quality and the comfortable treatment they can offer $[5,19]$.

Moreover, health and medical tourism industry has been in increasing growth in many countries, particularly in Asia, Latin America, United States, Canada, and Western Europe, where healthcare is getting costlier [15], or in South Africa and India from war-torn neighboring countries with poor health infrastructure for reducedwait time and/or high-quality medical care at reasonable costs [26]. This intense demand can affect the quality and the efficiency of healthcare services. To meet this problem, healthcare units usually concentrate on the optimization of their resources' utilization and minimizing total completion time, even more so in chemotherapy clinics where the number of cancer patients increased intensely [24]. Indeed, healthcare services are required to be performed by different resources such as chairs or beds injection, oncologists, phlebotomists or pharmacists and nurses. There are various ways to administrate Chemotherapy: intravenously, orally, through an injection, or topically (applied on the skin). The most common method of delivering chemotherapy is intravenously and the procedures range from $15 \mathrm{~min}$ to $7 \mathrm{~h}$ or longer. These variations complicate the patient's appointments schedule and resources allocation. Every patient is associated with a referee physician who ensures the follow-up of the care. Hence, a patient has to come the day when his referee physician works. The private sector is distinct by the fact that doctors are, unlike the public sector, independent service providers $[29,33]$. Consequently, another physician cannot see the patient. His own oncologist must examine him.

In this paper, we focus on daily scheduling appointments in an outpatient oncology department of a Tunisian private clinic. All patients receive chemotherapy by appointment according to their therapeutic planning which is fixed by his referent oncologist. Currently, the appointment specifies only the treatment day. Therefore, all patients come early in the morning with their blood tests results and wait for their oncologists to begin the treatments. The average duration of waiting time per day is $123.08 \mathrm{~min}$. Treatment lengths range from 15 to $4808 \mathrm{~min}$, with the most common appointment duration equal to $158 \mathrm{~min}$. The average day lasted $8.4 \mathrm{~h}$ or $504 \mathrm{~min}$. The outpatient chemotherapy department's service is supposed to provide services from 8:00 to 16:00 on weekdays. However, they usually exceed the expected completion time.

Our work consists in improving the quality of services to patients by providing an appointment schedule and assigning patients to all resources available at each stage while optimizing the makespan.

In general, the chemotherapy process is composed of three phases: consultation, drug's preparation and injection. As the injection is a very particular phase that should be decomposed into three successive tasks (connection, control, and disconnection), we have considered in this study the following decomposition into five stages (see Fig. 1):

- The first stage (consultation): consultation realized by a referent oncologist respecting a therapeutic planning.

- The second stage (drugs' preparation): after consultation, the oncologist prescribes the drugs' dose and a pharmacist prepares the drugs to administrate it by a nurse.

- The third stage (connection stage): when a chair is available, the patient is settled by a nurse who connects him/her after checking his/ her blood tests, weight and temperature.

- The fourth stage (control stage): the nurse must monitor his/her patient along the injection, she can take care of four patients at the same time.

- The fifth stage (disconnection stage): finally, the nurse disconnects him/her, examines him/her and provides instructions for home care and for the next appointment.

Each patient will go through these five stages. The waiting time between the first three stages will be minimized. However, the three last stages (stage 3, stage 4 and stage 5) composing the injection phase should be done successively without a waiting time between them. The paper is structured as below: Section 2 provides a brief overview of the chemotherapy appointment-scheduling problems in the literature. In Section 3, we give a description of the considered problem. In Section 4, we mathematically formulate the problem. We introduce then our lower bound in Section 5. In Section 6, we present a tabu search inspired (TS) algorithm to obtain 
a better solution especially for large instances. We lead an experimental study in Section 7 to evaluate the performance of our approaches by measuring the relative gaps between the returned solutions and the lower bound. Our conclusion and research perspectives are presented in Section 8.

\section{Literature REVIEW}

In the literature, many approaches have been developed to solve appointment-scheduling problems related to oncology clinics. Most of them can be classified into two major classes: exact approaches and approximate approaches. On one hand, numerous studies used exact optimization methods to schedule appointments by offering optimal solutions supporting issues of patient flow and resource exploitation [3,42]. Simulation and approximate methods, on the other hand, can handle well large and complex systems with difficult constraints $[13,37]$.

Mathematical programming is applied to improve scheduling process in chemotherapy clinics. Sadki et al. [32] developed an integer programming model to control oncologist and chemotherapy appointments with the objective of minimizing a weighted combination of patient waiting time and makespan (clinic total working time). They assumed that nurses have enough capacity and considered only the treatment stage. Condotta et al. [3] developed a multilevel template for patient appointment scheduling with the main objective of minimizing patients' waiting times and nurses' workload. Liang et al. [22] presented a multi-objective optimization model to solve the patient scheduling problem in a primary care delivery model (each patient is assigned to a primary nurse every time he/she comes to the clinic) with the objective of minimizing total overtime and total excess workload. Shashaani [35] developed an integer-programming model to determine the optimal appointment schedule that is used later as an input to a simulation model to estimate the impact of variability on patient waiting times. Benzaid et al. [2] dealt with patient-nurse assignment problem. They developed a mathematical formulation procedure to schedule treatments for new patients, to plan nurse requirements, and to assign the daily patient mix to available nurses. Huang et al. [18] developed a chemotherapy scheduling template. They account in their model many constraints like nurse resource availability and patient treatment needs. The objective of this study is to create a scheduling template minimizing the violation between resource assignment and treatment requirements. Huang et al. [17] presented an optimization model to determine when to schedule patients according to their visit durations. The model improves chair utilization and reduces staffing resource violations measured by nurseto-patient ratios during the workday. Hesaraki et al. [14] proposed an integer programming model to generate an arrangement of vacant appointment slots considering nursing constraints. The objective of the proposed model was to minimize makespan.

A vast literature on approximation methods has been developed in the last decade, especially for scheduling problems, Shahnawaz [36] proposes an efficient algorithm using a TS to schedule the identical parallel machines with single resource. Minh-Duc Le et al. [25] paper studies meta-heuristic methods in solving an appointmentscheduling problem in a hospital in Canada. Their initial solution was generated with a Greedy Algorithm. Then, they choose the best strategy to improve it with TS. Patients must be assigned to nurses with specific treatment seats and respecting the treatment protocol.

Turckan et al. [39] developed an integer-programming model to solve the deterministic chemotherapy outpatient-scheduling problem. For instances with large number of patients per day ( 50 patients), they developed a heuristic to solve the problem in a short computational time. The objective was to provide a nurse-patient assignment model considering patient acuity metrics and nurses' perceived workload.

Simulation is used to test the impact of different scheduling methods in order to identify the best approach. Tanaka [38] considered the time allocated for pre-treatment process, preparation and nursing, and used simulation to experiment different scheduling rules based on bin-packing algorithms. El-Mekkawy and Bates [1] and Yokouchi et al. [43] used simulation to define the best appointment scheduling rules. Many tests are executed to describe the impact of varying the number of nurses at each time interval. Santibáñez et al. [34] created a scheduling system for a large chemotherapy clinic in British Columbia using simulation and mixed integerprogramming models. The simulation addressed the problem of assigning a date to the first chemotherapy 
appointment for each patient to reduce the waiting list. The mixed integer-programming model is used, then, for daily scheduling to minimize the maximum nurse workload. Woodall et al. [41] used simulation based optimization to find the optimal nurse schedules with the objective of minimizing expected waiting times. Garaix et al. [7] proposed a heuristic approach to schedule a list of patients for three main phases: consultation, drug preparation and injection phases. Two objectives are considered; the closing time and the overworking time of the clinic.

We notice that the literature chemotherapy scheduling mostly focuses on one phase especially the injection phase and its human and material resources management. To the best of our knowledge, only one paper similarly considered three main phases [7]. However, they did not consider the same assumptions and assumed that an adequate number of nurses is always available where the nurse capacity is a fundamental constraint in our study. Moreover, they did not take into account connection and disconnection phases and they also considered that the pharmacy is not a bottleneck for drugs preparation. In this paper, we provide a mathematical model to solve the chemotherapy problem with the consideration of the three phases' resources constraints (see Fig. 1): oncologist visit, pharmacist drugs' preparation and chemotherapy treatment (or injection).

We believe that the contributions of this study are as below:

(1) The integration of all chemotherapy phases within the daily scheduling problem: To the best of our knowledge, there are no notable works in the literature that dealt with all the phases and considered related human and material resources and constraints during the three phases. The proposed study is the first optimization-based method that considers all chemotherapy phases with the decomposition of the injection phase to three sub-phases, and the related human and material resources.

(2) The multi-stage scheduling problem is known to be NP-hard [6]. We provide a MIP model and minimize the makespan. Since the mathematical problem could not solve large instances, we provided a lower bound expression and developed approximated solving algorithms: a constructive heuristic and an improvement procedure inspired from the tabu search approach that can offer near-optimal or good results in a reasonable computational time.

\section{Problem DESCRIPTION}

The chemotherapy outpatient-scheduling problem consists in sequencing a fixed number of patients and assigning them to resources, over a 1-day period, while considering consultation, drugs' preparation and injection phases.

According to Graham's notation [12], we can represent the scheduling problem of the chemotherapy unit in the Tunisian private hospital as an FF3:1,3,1;2,2,1;3,5(10),1/0.25(1) |prec,temp| Cmax problem [23], where the first group describes the machine environment, the second group defines the processing characteristics, and the third group presents the objectives.

In our study, we consider a special flexible flow-shop composed of three main phases (represented by FF3 in the problem notation). The first phase (consultation) has one type of machine (oncologist) with three available units, and each patient needs one unit, indicated in the notation by $1,3,1$. The second phase (pharmacy) has one type of machines (pharmacist), with two available units, and each patient requires one unit, represented in the notation by $2,2,1$. The third phase (injection) has one type of machines (nurses) and one type of additional resources (chairs or bed). This phase is particular and can be divided into three sub-phases: first, the patient occupies a chair, then the nurse starts by connecting the patient and checking his/her blood tests, after that, controls him/her along the drugs' administration and finally disconnects him/her, examines him/her and provides instructions for home care. For the controlling stage, one nurse can take care of maximum four patients at the same time. In addition, a nurse cannot connect or disconnect more than one patient at the same time. The nurse can start, for example, by connecting the first patient, then the second patient, after that the third and finally the fourth patient. Then, the nurse cannot connect a fifth patient before disconnecting one of the four connected patients to not monitor more than four patients at their injection phase. The nurse can connect or disconnect a patient and at the same time monitor other patients at their injection phase. In our case, five 


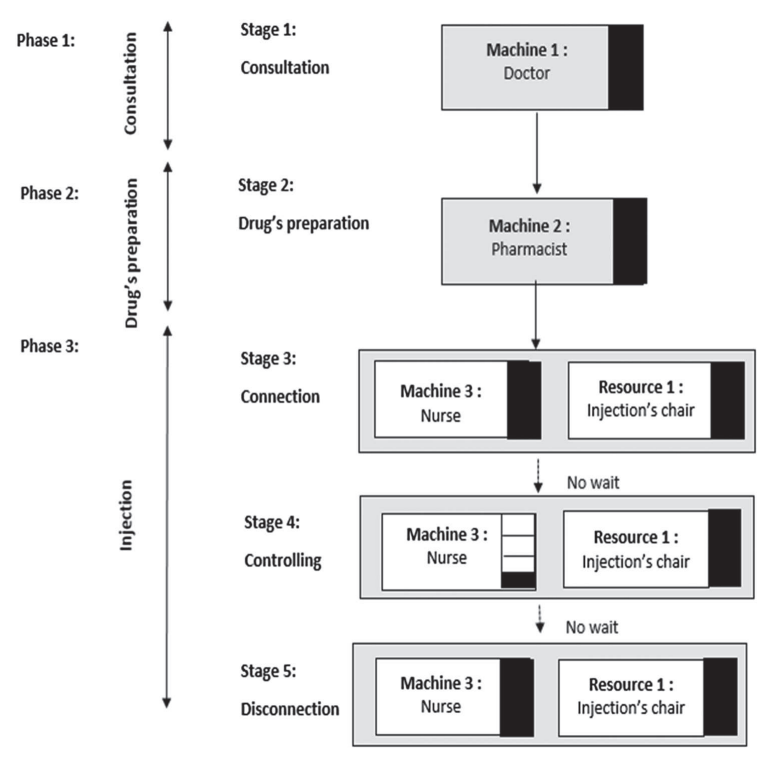

Figure 1. Problem description.

nurses and ten chairs are available represented in the notation by $3,5(10), 1 / 0.25(1)$. The third phase with five nurses and ten chairs, each patient requires one nurse during the connection/disconnection sub-phase and 0.25 nurse during the control sub-phase (as a nurse can simultaneously control up to 4 patients). In addition to the nurse, the patient requires one chair during the whole third phase.

There are two types of patients: the follow-up patients and the new patients coming to receive treatment for the first time. New patients need more time for the consultation and the nurse set-up. Treatment durations can last from $158 \mathrm{~min}$ to $8 \mathrm{~h}$. The process characteristics include precedence constraints and in certain cases no-wait constraints between stages; represented in the notation by prec and temp. The objective is to minimize the makespan (i.e. the ending time of the last completed operation), which is presented in the notation by Cmax. However, there are many other performance criteria to be considered when solving scheduling problems. We choose this criterion because, especially for private sector, when the main objective is to increase profits, it is very important to maximize the number of admitted patients. In fact, by minimizing completion time, we maximize patient admission while increasing the usage of human resources and medical equipment [11].

The motivation of this study grew out with the significant increasing number of patients per day and the importance of providing an optimal scheduling to improve the number of admitted patients in the clinic and to optimize delaying treatment, which could have negative effects on the patient $[5,23,28]$. Therefore, minimizing the time needed to complete all the treatment process, including all stages, can be very important.

This problem can be presented, in our case, as a five-stage hybrid flow shop-scheduling problem that uses four different resources (see Fig. 1): oncologists, pharmacists, nurses and injection chairs. We present an example (see Tab. 1 and Fig. 2) where a small sized problem is provided to illustrate the problem ( 5 min slot time). We considered, in this example, 10 patients, 5 chairs, 3 oncologists, 2 nurses and 2 pharmacists. All stages durations are detailed in Table 1 (minutes).

This research aims to model and solve the chemotherapy scheduling problem. We develop a mathematical model and solving techniques to handle the chemotherapy-scheduling problem by optimally assigning patients to oncologists, pharmacists, nurses and chairs. The special feature of this problem is to align constraints from well-known scheduling problems: the parallel machine scheduling and the hybrid flow-shop scheduling problems which, mathematically, has been proved to be NP-hard. In fact, the HFSP is, in most cases, NP-hard [12]. 
TABLE 1. Example 1: Patient's stages duration.

\begin{tabular}{llllll}
\hline \hline (min) & $\begin{array}{l}\text { Consul. } \\
(\mathrm{min})\end{array}$ & $\begin{array}{l}\text { Drugs preparation } \\
(\mathrm{min})\end{array}$ & $\begin{array}{l}\text { Connection } \\
(\mathrm{min})\end{array}$ & $\begin{array}{l}\text { Injection } \\
(\mathrm{min})\end{array}$ & Disconnection \\
\hline P1 & 10 & 5 & 5 & 25 & 5 \\
P2 & 10 & 15 & 5 & 15 & 5 \\
P3 & 20 & 5 & 5 & 45 & 5 \\
P4 & 10 & 5 & 10 & 45 & 5 \\
P5 & 10 & 35 & 5 & 5 & 5 \\
P6 & 5 & 5 & 5 & 50 & 5 \\
P7 & 15 & 10 & 5 & 50 & 5 \\
P8 & 10 & 5 & 5 & 20 & 5 \\
P9 & 5 & 35 & 5 & 5 & 5 \\
P10 & 15 & 5 & 5 & 5 & 5 \\
\hline
\end{tabular}

Even in the case when one stage includes two machines and the other one only a single machine. Similarly, even when machines can stop processing operations before their completion, it results also in strongly NP-hard problems, according to Hoogeveen et al. [16]. Moreover, the special case where there is a single machine per stage, known as the flow shop, and the case where there is a single stage with several machines, known as the parallel machines' environment, are also NP-hard [31].

\section{Mathematical Formulation}

In this section, we formally present the problem by providing a mathematical model with related data and sets:

\section{Data}

$P \quad$ Number of patients $(p=1, \ldots, P)$.

$O \quad$ Number of oncologists $(o=1, \ldots, O)$.

$N \quad$ Number of nurses $(n=1, \ldots, N)$.

$\mathrm{Ph} \quad$ Number of pharmacists $(\mathrm{ph}=1, \ldots, \mathrm{Ph})$.

$C \quad$ Number of chairs $(c=1, \ldots, C)$.

$S \quad$ Number of slots on one day $(s=1, \ldots, S)$.

Preaf $_{p} \quad$ Referee oncologist of patient $p$.

$\operatorname{Dur}_{i, p} \quad$ Processing time of stage $i$ of patient $p$ (in number of slots).

$M \quad$ A big number

\section{Sets}

$W_{p, o, s} \quad 1$ if the consultation of patient $p$ begins to occupy oncologist $o$ at time slot $s, 0$ otherwise.

$Z_{p, \mathrm{ph}, s} \quad 1$ if pharmacist ph begins the drugs preparation of patient $p$ at time slot $s, 0$ otherwise.

$X_{p, c, s} \quad 1$ if patient $p$ begins the injection phase on chair $c$ at time slot $s, 0$ otherwise.

$Y_{p, n, s} \quad 1$ if patient $p$ begins to occupy the nurse $n$ at time slot $s, 0$ otherwise.

$\mathrm{VT}_{p, n, s} 1$ if nurse $n$ is occupied by the connection, the injection or the disconnection of patient $p$ at time slot $s, 0$ otherwise.

$V 1_{p, n, s} \quad 1$ if nurse $n$ at time slot $s$ is occupied by the connection of a patient, 0 otherwise. 
Doctors planning

\begin{tabular}{|c|c|c|c|c|c|c|c|c|c|c|c|c|c|c|c|c|c|c|c|c|c|c|}
\hline & 1 & 2 & 3 & 4 & 5 & 6 & 7 & 8 & 9 & 10 & 11 & 12 & 13 & 14 & 15 & 16 & 17 & 18 & 19 & 20 & 21 & 22 \\
\hline D1 & P7 & P7 & P7 & P2 & P2 & & & P4 & P4 & & & & & & & & & & & & & \\
D2 & P8 & P8 & P6 & & & & & & P5 & P5 & & & & & & & & & & & & \\
D3 & P1 & P1 & P10 & P10 & P10 & P3 & P3 & P3 & P3 & P9 & & & & & & & & & & & & \\
\hline
\end{tabular}

Pharmacists planning

\begin{tabular}{|l|l|c|c|c|c|c|c|c|c|c|c|c|c|c|c|c|c|c|c|c|c|c|}
\hline & 1 & 2 & 3 & 4 & 5 & 6 & 7 & 8 & 9 & 10 & 11 & 12 & 13 & 14 & 15 & 16 & 17 & 18 & 19 & 20 & 21 & 22 \\
\hline Ph1 & & & P1 & P7 & P7 & P10 & & & & P4 & P9 & P9 & P9 & P9 & P9 & P9 & P9 & & & & & \\
Ph2 & & & P8 & P6 & & P2 & P2 & P2 & & P3 & P5 & P5 & P5 & P5 & P5 & P5 & P5 & & & & & \\
\hline
\end{tabular}

Chairs planning

\begin{tabular}{|c|c|c|c|c|c|c|c|c|c|c|c|c|c|c|c|c|c|c|c|c|c|c|}
\hline & 1 & 2 & 3 & 4 & 5 & 6 & 7 & 8 & 9 & 10 & 11 & 12 & 13 & 14 & 15 & 16 & 17 & 18 & 19 & 20 & 21 & 22 \\
\hline Ch1 & & & & P8 & P8 & P8 & P8 & P8 & P8 & & P4 & P4 & P4 & P4 & P4 & P4 & P4 & P4 & P4 & P4 & P4 & P4 \\
\hline Ch2 & & & & & & & P10 & P10 & P10 & P2 & P2 & P2 & P2 & P2 & & & & P9 & P9 & P9 & & \\
\hline Ch3 & & & & & P6 & P6 & P6 & P6 & P6 & P6 & P6 & P6 & P6 & P6 & P6 & P6 & & & & & & \\
\hline Ch4 & & & & P1 & $\mathrm{P} 1$ & P1 & P1 & P1 & $\mathrm{P} 1$ & P1 & P3 & P3 & P3 & P3 & P3 & P3 & P3 & P3 & P3 & P3 & P3 & \\
\hline Ch5 & & & & & & P7 & P7 & P7 & P7 & P7 & P7 & P7 & P7 & P7 & P7 & P7 & P7 & & P5 & P5 & P5 & \\
\hline
\end{tabular}

Patients planning
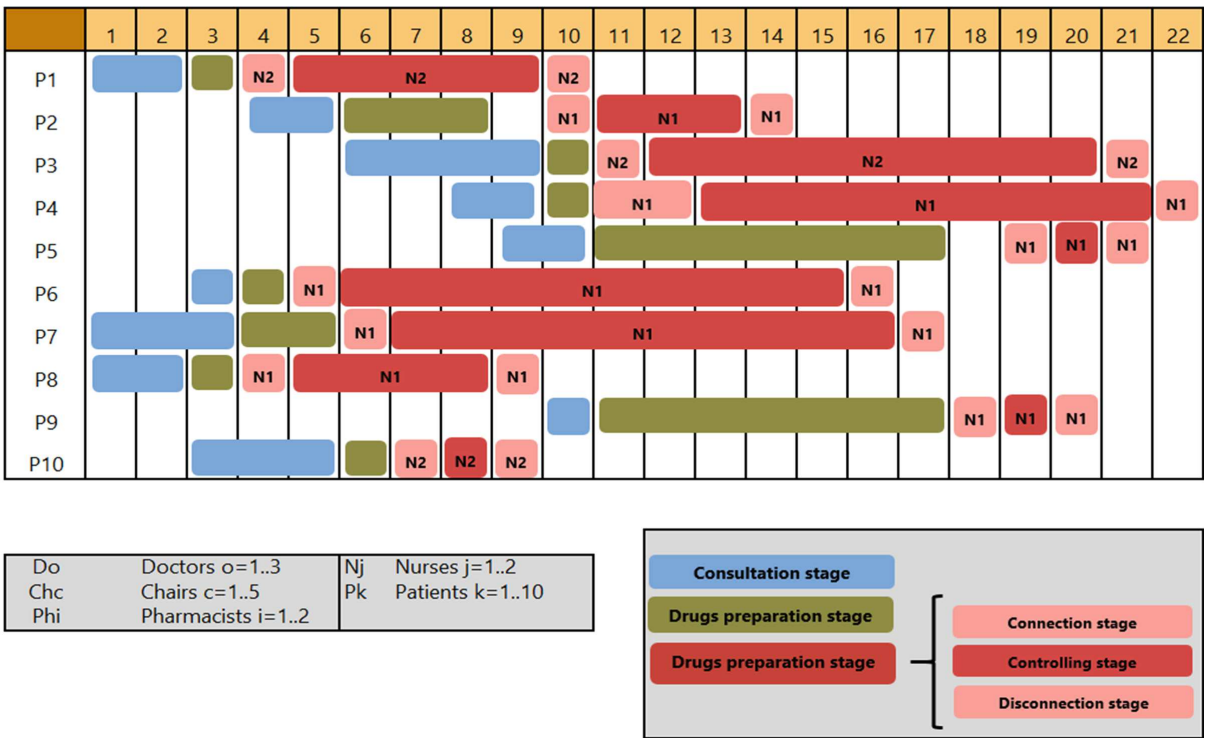

Figure 2. Scheduling template of 10 patients with 3 oncologists, 2 pharmacists, 5 chairs and 2 nurses. 
$V 3_{p, n, s}$

1 if nurse $n$ at time slot $s$ is occupied by the disconnection of a patient, 0 otherwise.

Chargepr $1_{n, s}$

Charge $_{n, s}$

Number of patients being connected by nurse $n$ at time slot $s$.

Chargepr $2_{n, s}$

$\mathrm{Dc}_{p}$

$\mathrm{Dp}_{p}$

$\mathrm{Di}_{p}$

$C_{\max }$

Number of patients monitored by nurse $n$ at time slot $s$.

Number of patients being disconnected by nurse $n$ at time slot $s$.

Starting time of patient $p$ for medical consultation.

Starting time of patient $p$ for drugs preparation.

Injection starting time of patient $p$ (set-up starting time of patient $p$ ).

Maximum completion time of all patients in the last stage.

Using these definitions, the studied problem can be mathematically formulated as below:

Obj: $\min C_{\max }$

subject to

$$
\begin{aligned}
& M\left(W_{p, o, s}-1\right)+\sum_{p^{\prime}=1}^{P} \sum_{t=s}^{s+\operatorname{Dur}_{1, p}-1} W_{p^{\prime}, o, t} \leq 1 \quad \forall p \in\{1, \ldots, P\}, \forall s \in\{1, \ldots, S\}, \\
& \sum_{s=1}^{S} W_{p, \operatorname{Preaf}_{p}, s}=1 \\
& \forall o \in\{1, \ldots, O\} \\
& \forall p \in\{1, \ldots, P\} \\
& \mathrm{Dc}_{p}=\sum_{s=1}^{S} W_{p, \operatorname{Preaf}_{p}, s} * S \\
& \forall p \in\{1, \ldots, P\} \\
& \sum_{s=1}^{S} \sum_{\mathrm{ph}=1}^{\mathrm{Ph}} Z_{p, \mathrm{ph}, s}=1 \\
& \forall p \in\{1, \ldots, P\} \\
& M\left(Z_{p, \mathrm{ph}, s}-1\right)+\sum_{p^{\prime}=1}^{P} \sum_{t=s}^{s+\operatorname{Dur}_{2, p}-1} Z_{p^{\prime}, \mathrm{ph}, t} \leq 1 \quad \forall p \in\{1, \ldots, P\}, \forall s \in\{1, \ldots, S\}, \\
& \forall \mathrm{ph} \in\{1, \ldots, \mathrm{Ph}\} \\
& \mathrm{Dp}_{p}=\sum_{s=1}^{S} \sum_{\mathrm{ph}=1}^{\mathrm{Ph}} Z_{p, \mathrm{ph}, s} * S \\
& \forall p \in\{1, \ldots, P\} \\
& M\left(X_{p, c, s}-1\right)+\sum_{p^{\prime}=1}^{P} \sum_{t=s}^{s+\operatorname{Dur}_{3, p}+\operatorname{Dur}_{4, p}+\operatorname{Dur}_{5, p}-1} \\
& \times X_{p^{\prime}, c, t} \leq 1 \\
& \forall p \in\{1, \ldots, P\}, \\
& \forall s \in\{1, \ldots, S\}, \forall c \in\{1, \ldots, C\} \\
& \sum_{s=1}^{S} \sum_{c=1}^{C} X_{p, c, s}=1 \\
& \forall p \in\{1, \ldots, P\} \\
& \operatorname{Di}_{p}=\sum_{s=1}^{S} \sum_{c=1}^{C} X_{p, c, s} * S \quad \forall p \in\{1, \ldots, P\} \\
& \sum_{n=1}^{N} Y_{p, n, s}=\sum_{c=1}^{C} X_{p, c, s} \quad \forall p \in\{1, \ldots, P\}, \forall s \in\{1, \ldots, S\} \\
& \operatorname{Di}_{p}+\sum_{i=3}^{5} \operatorname{Dur}_{i, p} \leq C_{\text {max }} \quad \forall p \in\{1, \ldots, P\}
\end{aligned}
$$


$\mathrm{VT}_{p, n, t} \geq Y_{p, n, s}$

$\sum_{s=1}^{S} \mathrm{VT}_{p, n, s}=\sum_{i=3}^{5} \operatorname{Dur}_{i, p}$

Charge $_{n, s}=\sum_{p=1}^{P} \operatorname{VT}_{p, n, s}$

$V 1_{p, n, t} \geq Y_{p, n, s}$

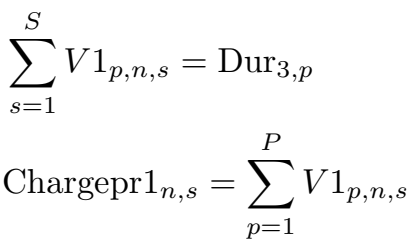

$V 3_{p, n, t} \geq Y_{p, n, s}$

$\sum_{s=1}^{S} V 3_{p, n, s}=\operatorname{Dur}_{5, p}$

Chargepr $2_{n, s}=\sum_{p=1}^{P} V 3_{p, n, s}$

Charge $_{n, s} \leq 4$

Chargepr $1_{n, s}+$ Chargepr $2_{n, s} \leq 1$

$\mathrm{Dc}_{p}+\operatorname{Dur}_{1, p} \leq \mathrm{Dp}_{p}$

$\mathrm{Dp}_{p}+\operatorname{Dur}_{2, p} \leq \mathrm{Di}_{p}$ $\forall p \in\{1, \ldots, P\}, \forall s \in\{1, \ldots, S\}, \forall n \in\{1, \ldots, N\}$,

$\forall t \in\left\{s, \ldots, s+\sum_{i=3}^{5} \operatorname{Dur}_{i}, p-1\right\}$

$\forall p \in\{1, \ldots, P\}, \forall n \in\{1, \ldots, N\}$

$\forall n \in\{1, \ldots, N\}, \forall s \in\{1, \ldots, S\}$

$\forall p \in\{1, \ldots, P\}, \forall s \in\{1, \ldots, S\}, \forall n \in\{1, \ldots, N\}$,

$\forall t \in\left\{s, \ldots, s+\operatorname{Dur}_{3, p}-1\right\}$

$\forall p \in\{1, \ldots, P\}, \forall n \in\{1, \ldots, N\}$

$\forall n \in\{1, \ldots, N\}, \forall s \in\{1, \ldots, S\}$

$\forall p \in\{1, \ldots, P\}, \forall s \in\{1, \ldots, S\}, \forall n \in\{1, \ldots, N\}$

$\forall t \in\left\{s+\sum_{i=3}^{4} \operatorname{Dur}_{i, p}, \ldots, s+\sum_{i=3}^{5} \operatorname{Dur}_{i, p}-1\right\}$

$\forall p \in\{1, \ldots, P\}, \forall n \in\{1, \ldots, N\}$

$\forall n \in\{1, \ldots, N\}, \forall s \in\{1, \ldots, S\}$

$\forall n \in\{1, \ldots, N\}, \forall s \in\{1, \ldots, S\}$

$\forall n \in\{1, \ldots, N\}, \forall s \in\{1, \ldots, S\}$

$\forall p \in\{1, \ldots, P\}$

$\forall p \in\{1, \ldots, P\}$.

Expression (4.1) describes the objective function, which consists of minimizing the total completion time (makespan). Constraints (4.2) ensure that an oncologist has a single patient during his/her consultation time. Equations (4.3) guarantee that a patient had a single medical consultation with his/her referee oncologist during the whole horizon. Constraints (4.4) calculate the starting time of patient's consultation. Equations (4.5) reflect that each patient is assigned to one pharmacist and once during the whole horizon. Constraints (4.6) ensure that each pharmacist at each slot prepare one patient's drugs i.e. he cannot prepare drugs for two patients at the same time. Equations (4.7) calculate the starting time for preparing drugs of patient $p$. Constraints (4.8) guarantee that at most a single patient occupies a chair during his/her injection time. Constraints (4.9) ensure that each patient is scheduled for injection only once during the whole horizon and to one chair. Constraints (4.10) calculate the starting time of patient's injection. Equations (4.11) guarantee that a patient starts to occupy a chair and a nurse for injection at the same time. Constraints (4.12) ensure that the makespan is greater than or equal to the ending date of each operation. Constraints (4.13) force the occupation binary variable $\mathrm{VT}_{p, n, s}$ to 1 (during the last three stages) if nurse $n$ starts to take charge of patient $p$ at time slot $s$. Constraints (4.14) state that a nurse $n$ remains occupied by a patient $p$ during the whole processing time at stages 3,4 and 5 . Constraints (4.15) calculate the number of patients controlled by nurse $n$ during a time slot $s$. Constraints 
(4.16) force the binary variable $V 1_{p, n, s}$ to 1 (during the connection stage) if nurse $n$ starts the connection of patient $p$ at time slot $s$. Constraints (4.17) state that a nurse is considered as connecting a patient during the whole processing time at stage 3. Constraints (4.18) define the number of patients connected by nurse $n$ during time slot $s$. Constraints (4.19) force the binary variable $V 3_{p, n, s}$ to 1 (during the disconnection stage) if nurse $n$ starts the disconnection of patient $p$ at time slot $s$. Constraints (4.20) state that a nurse is considered as disconnecting a patient during the whole processing time at stage 5. Constraints (4.21) determine the number of patients disconnected by a nurse $n$ at a given time slot. Constraints (4.22) notify that a nurse can control at most four patients at the same time. Constraints (4.23) ensure that a nurse connects or disconnects at most one patient in any time slot s. Constraints (4.24) confirm that drug's preparation starts after oncologist consultation. Constraints (4.25) guarantee that drugs administration starts when the drugs are ready.

In the considered problem, if the number of stages is equal to five, the number of machines in each stage is equal to one and capacity for all machines are equal to one, our problem is converted to F5//Cmax which its time complexity functions is known to be NP-hard (Garey et al., [8]). In the last three stages, we consider two types of resources the nurses and the chairs.

Therefore, the time complexity function of HFSP is at least NP-hard, which reflects that finding an optimum solution to the problem in a reasonable time is unlikely and hence heuristic approaches should be employed.

\section{LOWER BOUND}

We found several lower bounds in the literature adapted to hybrid flow shop scheduling problems.

Oğuz et al. [27] proposed several lower bounds for a two-stage Hybrid flow shop scheduling with multiprocessor task with arbitrary processing time. Wang et al. [40] proposed a mixed integer programming model and derive a tighter lower bound for the same case. Li et al. [21] utilized a lower bound based on construction algorithm, they used the proposed lower bound in a branch-and bound algorithm to solve HFSP and study how rescheduling affects surgeons' delays and overtime. Ying and Lin [42] provided a lower bound and considered that for each stage, there are only machines and no additional resources.

Our problem presents some specific constraints such as resource constraints and no-waiting constraints in the injection phase. Thus, we provide a new lower bound for our specific problem. We got inspired by the lower bound proposed in $[31,42]$.

To calculate the lower bound LB for our problem, we considered the maximum between two values LB1 and LB2. The first term LB1 is a lower bound based on jobs (patients), and the second term LB2 is a lower bound based on stages

$$
\mathrm{LB}=\max (\mathrm{LB} 1, \mathrm{LB} 2) .
$$

We first calculate LB1, which is based on the processing time of the patient having the longest duration:

$$
\mathrm{LB1}=\max _{p=1, \ldots, P}\left(\sum_{i=1}^{5} \operatorname{Dur}_{i, p}\right)
$$

where: $P$ is the number of patients and $\operatorname{Dur}_{i, p}$ presents the duration that a patient $p$ spends on stage $i(i=$ $1, \ldots, 5)$.

The second value LB2 is calculated as the maximum value of the lower bounds of all stages $i:(i=1, \ldots, 5)$ :

$$
\mathrm{LB} 2=\max _{i=1, \ldots, 5}\left(\mathrm{LBS}_{i}\right) .
$$

To determine precisely the value of $\mathrm{LBS}_{i}$, we assume that the scheduling is ensured without delay. Under this hypothesis, the time necessary to start the execution of the operation of a patient $p=1, \ldots, P$, on any machine of the considered stage $i$, is at best equal to $\min _{p=1, \ldots, P}\left(\sum_{l=1}^{i-1} \operatorname{Dur}_{l, p}\right)$. Similarly, the time required to complete the execution of jobs on the remaining stages (from $i+1$ to 5 ) is $\min _{p=1, \ldots, P}\left(\sum_{l=i+1}^{5} \operatorname{Dur}_{l, p}\right)$. 
It remains to evaluate a limit on the duration of patient's treatment on stage $i$. It is the maximum between two values: The first value is presented by the sum of the durations of all operations on stage $i$ divided by $n m_{i}$ which describes the number of machines available at that stage: $\sum_{p=1}^{P} \operatorname{Dur}_{i, p} / n m_{i}$. The second value corresponds to the maximum duration of the considered stage: $\max _{p \in P}\left(\operatorname{Dur}_{i, p}\right)$. Thus, the value of $\mathrm{LBS}_{i}$ for consultation stage and preparation stage is:

$$
\begin{aligned}
& \operatorname{LBS}_{1}=\max \left(\max _{p \in P}\left(\operatorname{Dur}_{1, p}\right), \sum_{p=1}^{P} \frac{\operatorname{Dur}_{1, p}}{n m_{1}}\right)+\min _{p=1, \ldots, P}\left(\sum_{l=2}^{5} \operatorname{Dur}_{l, p}\right) \\
& \mathrm{LBS}_{2}=\min _{p=1, \ldots, P}\left(\operatorname{Dur}_{1, p}\right)+\max \left(\max _{p=1, \ldots, P}\left(\operatorname{Dur}_{2, p}\right), \sum_{p=1}^{P} \frac{\operatorname{Dur}_{2, p}}{n m_{2}}\right)+\min _{p=1, \ldots, P}\left(\sum_{l=3}^{5} \operatorname{Dur}_{l, p}\right) .
\end{aligned}
$$

For stages with additional resources (stage 3,4 and 5), we must add a third value, this value is calculated by dividing the sum of the durations of all operations on stage $i$ by $n r_{i}$ which describes the number of resources available at this stage. The value of $\mathrm{LBS}_{i}$ in this case is as follow:

$$
\begin{aligned}
\mathrm{LBS}_{3}= & \min _{p=1, \ldots, P}\left(\sum_{l=1}^{2} \operatorname{Dur}_{l, p}\right)+\max \left(\max _{p=1, \ldots, P}\left(\operatorname{Dur}_{3, p}\right), \sum_{p=1}^{P} \frac{\operatorname{Dur}_{3, p}}{n m_{3}}, \sum_{p=1}^{P} \frac{\operatorname{Dur}_{3, p}}{n r_{3}}\right) \\
& +\min _{p=1, \ldots, P}\left(\sum_{l=4}^{5} \operatorname{Dur}_{l, p}\right) \\
\mathrm{LBS}_{4}= & \min _{p=1, \ldots, P}\left(\sum_{l=1}^{3} \operatorname{Dur}_{l, p}\right)+\max \left(\max _{p=1, \ldots, P}\left(\operatorname{Dur}_{4, p}\right), \sum_{p=1}^{P} \frac{\operatorname{Dur}_{4, p}}{n m_{4}}, \sum_{p=1}^{P} \frac{\operatorname{Dur}_{4, p}}{4 * n r_{4}}\right) \\
& +\min _{p=1, \ldots, P}\left(\operatorname{Dur}_{5, p}\right) \\
\mathrm{LBS}_{5}= & \min _{p=1, \ldots, P}\left(\sum_{l=1}^{4} \operatorname{Dur}_{l, p}\right)+\max \left(\max _{p=1, \ldots, P}\left(\operatorname{Dur}_{5, p}\right), \sum_{p=1}^{P} \frac{\operatorname{Dur}_{5, p}}{n m_{5}}, \sum_{p=1}^{P} \frac{\operatorname{Dur}_{5, p}}{n r_{5}}\right) .
\end{aligned}
$$

LB2 determines the maximum of lower bound over all stages $i=1, \ldots, 5$, assuming no waiting between the stages. For each stage $i$, the expression $\mathrm{LBS}_{i}$ is calculated by considering a single-stage multiprocessor task problem and adding the lower bound for the preceding stages and the succeeding stages. For the last three stages $i=3,4$ and 5 , we consider both machines (chairs: $n m_{i}$ ) and resources (nurses: $n r_{i}$ ).

The analysis of this lower bound shows that when the operations' durations in each stage are relatively close to each other, the bound LB is usually given by the value of LB2.

We can also notice that the more the processing times of the operations are small, the more the lower bound LB is close to the optimal solution. Indeed, for each stage, the value of $\mathrm{LBS}_{i}$ is obtained by equally dividing the durations of the operations among the machines in each stage; when some of these processing times are large, it becomes more difficult to find a scheduling that satisfies such a fair division, especially considering the precedence constraints between the operations using the same resource.

\section{Approximated Algorithm}

The Tabu Search (TS), proposed by Glover [9], is a meta-heuristic method that has been commonly used in combinatorial optimization. The general idea of this method is to compare at each iteration, a set of solutions from the neighborhood of the current one (Laguna et al., [20]), and moves to the best one among them [9]. At each iteration, a flexible memory maintains a list of banned moves (tabu list) to avoid being stuck in a local optimality. The global structure of our TS implementation is represented in Algorithm 2. 
In this paper, we present a constructive heuristic and an improvement procedure inspired from the tabu search method. The algorithm starts with the initial solution obtained by the constructive heuristic (Algorithm 1: H1) which is based on a priority order and the first come first served (FCFS) rules. In Algorithm 1, and to simplify the problem, stages 3-5 (i.e. the connection, injection and disconnection phases) are considered as one stage during which a patient occupies one chair and is taken care by the same nurse (i.e. a patient stays on the same chair and is monitored by the same nurse during the connection, injection and disconnection phases). A nurse can control up to four patients at the same time, but only one of them can be in the connection or disconnection phase. Since we have three stages $(j=1, \ldots, 3)$, we provide to each patient $(p)$ a priority degree (denoted by Prio $_{p}$ ) on each stage. In the first stage, this priority index is calculated on the basis of the patients' durations on the three stages. For the second and the third stages, we update the priority degree of each patient considering the ending time of the previous stage (see Algorithm 1). At each of the three stages, jobs (patients) are sorted in the decreasing order of their priority degrees (see Algorithm 1) then assigned one by one to the first available machine: oncologist (o) for consultation stage (in this first stage, each patient is assigned to his referent oncologist), pharmacist (ph) for drugs' preparation stage and nurse $(n)$ and chair $(c)$ for injection stage. In the latter stage, the patient is assigned to the first available nurse that can perform the connection, injection and disconnection phases without interruption (i.e. no idle time is allowed between these three phases of the injection stage) while respecting the capacity constraints. Technically, the algorithm creates a time window for each nurse. This window specifies how many patients a nurse is dealing with during time slot $s$ and indicates if one of them is in the connection or disconnection phase, which makes it easy to find the suitable nurse.

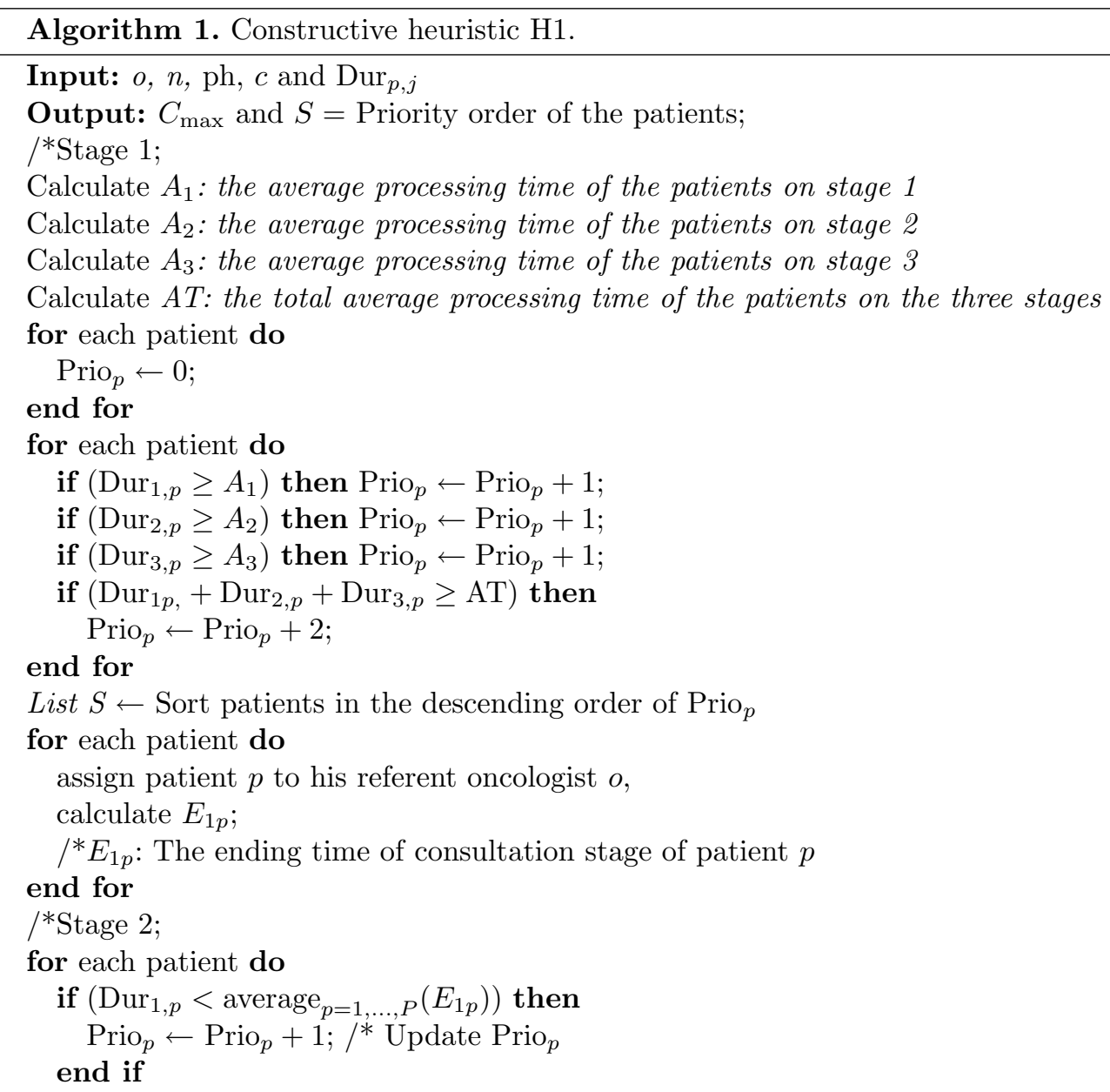




\section{end for}

Sort patients in the descending order of $\operatorname{Prio}_{p}$;

for each patient do assign patient $p$ to the first available pharmacist ph, calculate $E_{2 p}$;

${ }^{*} E_{2 p}$ : The ending time of drugs' preparation phase of patient $p$

\section{end for}

/*Phase 3 ;

for each patient do

$$
\begin{aligned}
& \text { if }\left(E_{2 p}<\operatorname{average}_{p=1, \ldots, P}\left(E_{2 p}\right)\right) \text { then } \\
& \text { Prio }_{p} \leftarrow \operatorname{Prio}_{p}+1 ; / * \text { Update Prio }_{p} \\
& \text { end if } \\
& \text { end for }
\end{aligned}
$$

Sort patients in the descending order of $\mathrm{Prio}_{p}$;

for each patient do

Assign patient $p$ to the first available chair $c$ and to the first available nurse $n$ that respects the no-wait and capacity constraints;

calculate $E_{3 p}$

$/^{*} E_{3 p}$ : The ending time of the disconnection phase of patient $p$

$$
\begin{aligned}
& \text { end for } \\
& \quad C_{\max } \leftarrow \max _{p=1, \ldots, P}\left(E_{3 p}\right) \\
& \quad \operatorname{return}\left(C_{\max }, S\right)
\end{aligned}
$$

The initial feasible solution $S$ from which the improvement procedure (Algorithm 2) starts its searching process is the one returned by our constructive heuristic. This algorithm explores the neighborhood of the current solution by randomly exchanging the positions of two patients in the priority list of stage 1 (the initial list is obtained from the first stage of Algorithm 1). A tabu list (size 10) is used to prohibit the reverse move during a certain number of iterations. If the solution does not improve after 10 iterations, a diversification procedure is used by randomly exchanging two patients in the priority order of stage 2. However, the beginning of a patient's treatment on stage 2 still has to wait until his consultation on stage 1 is over. The algorithm stops when a specified number of schedules are generated without improving the current solution. When this termination criterion is met (250 iterations without improving the solution), the algorithm returns the best solution found $\left(S^{*}\right)$.

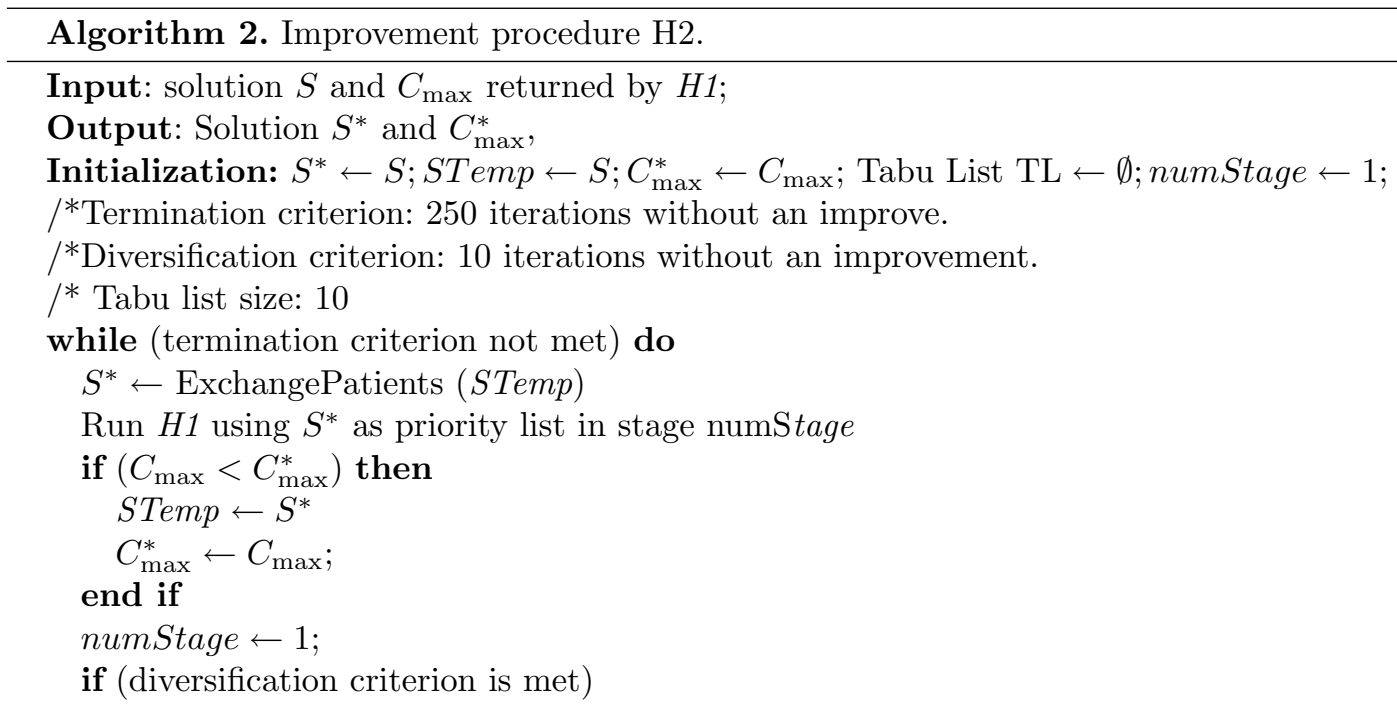


STemp $\leftarrow$ The priority order used in stage 2 in the last run of $H 1$

numStage $\leftarrow 2$;

\section{end while}

return $\left(C_{\max }^{*}, S T e m p\right)$

ExchangePatients $(S)$

\{

$S 1 \leftarrow$ exchange the positions of two patients in the priority order $S$

while $(S$ in TL)

$S 1 \leftarrow$ exchange the positions of two patients in the priority order $S$

end while

insert $S$ in TL

return $(S 1)$

\}

We applied the same example presented in Section 3 (Tab. 1) on heuristic H1 and we obtained a $C_{\max }$ equal to $150 \mathrm{~min}$. The corresponding schedule is represented on (Fig. 3). We then applied the improvement procedure $\mathrm{H} 2$ where we improved the result from a $C_{\max }$ equal to 150 to a $C_{\max }$ equal to $115 \mathrm{~min}$ (Fig. 4). The lower bound for this example is equal to $85 \mathrm{~min}$.

\section{EXPERIMENTAL STUDY}

To evaluate the performance of the proposed approaches, we conducted experiments on a set of randomly generated instances based on observations in a Tunisian clinic. The mathematical model was solved using CPLEX 12.5.1 solver with the default setting. The heuristic and the metaheuristic are coded with $\mathrm{C}++$ and the tests were held on a $16 \mathrm{~GB}$ computer with an Intel Xeon $3.00 \mathrm{GHz}$ processor.

\subsection{Generation of instances}

The generation of instances is based on the observation of more than 20 days (March 2016) from the daily scheduling process in a Tunisian clinic. We noted that the average number of appointments per day was 38.76 requests. This reflects the real-world context of Clinique Ennasr. Indeed, the clinic, as a private facility, collaborates with various oncologists of different specialties. In addition, the duration of the treatment depends on the nature of the cancer and fixed by the oncologist.

The probability distributions of the different durations were determined based on the collected data and summarized in Table 2. We determined the probability distribution that best fit the collected data using XLSTAT software.

We considered 10 different size problems from 5 patients to 50 patients with a step of 5 . For each size, we generated 10 instances (100 instances in total). Injection durations are generated according to a Lognormal probability distribution with an average of $\mu=150$ and a standard deviation $\sigma=82.1$. The rest of processing times are generated according to an exponential distribution with rate $\lambda$ (Tab. 2). Three oncologists and two pharmacists were considered for all instances. The referent oncologist is generated according to a normal distribution between 1 and 3. For 5 patients instances, we considered 2 nurses and 3 chairs. Starting for 10 patients, we increased the number of nurses to 5 with 10 chairs.

\subsection{Computational results}

We applied our mathematical model and both heuristics on the set of 100 generated instances. We considered a slot time of $5 \mathrm{~min}$ for all instances. For the mathematical model, we obtained the optimal solution for all instances with less than or equal to 10 patients. For 15 patients, we ran the mathematical model for $3 \mathrm{~h}$ and we noted the best solution obtained so far along with the best lower bound. For instances with 20 patients, we could not obtain solutions after running the model for more than $24 \mathrm{~h}$. 
Doctors planning

\begin{tabular}{|c|c|c|c|c|c|c|c|c|c|c|c|c|c|c|c|c|c|c|c|c|c|c|}
\hline & 1 & 2 & 3 & 4 & 5 & 6 & 7 & 8 & 9 & 10 & 11 & 12 & 13 & 14 & 15 & 16 & 17 & 18 & 19 & 20 & 21 & 22 \\
\hline D1 & P7 & P7 & P7 & P4 & P4 & P2 & P2 & & & & & & & & & & & & & & & \\
D2 & P6 & P5 & P5 & P8 & P8 & & & & & & & & & & & & & & & & & \\
D3 & P3 & P3 & P3 & P3 & P9 & P10 & P10 & P10 & P1 & P1 & & & & & & & & & & & & \\
\hline
\end{tabular}

Pharmacists planning

\begin{tabular}{|l|l|l|l|l|l|l|l|l|l|l|l|l|l|l|l|l|l|l|l|l|l|l|}
\hline & 1 & 2 & 3 & 4 & 5 & 6 & 7 & 8 & 9 & 10 & 11 & 12 & 13 & 14 & 15 & 16 & 17 & 18 & 19 & 20 & 21 & 22 \\
\hline Ph1 & & & & & $P 3$ & $P 4$ & $P 5$ & $P 5$ & $P 5$ & $P 5$ & $P 5$ & $P 5$ & $P 5$ & $P 2$ & $P 2$ & $P 2$ & & & & & & \\
Ph2 & & & & $P 7$ & $P 7$ & $P 6$ & $P 9$ & $P 9$ & $P 9$ & $P 9$ & $P 9$ & $P 9$ & $P 9$ & $P 10$ & $P 8$ & $P 1$ & & & & & & \\
\hline
\end{tabular}

Chairs planning

\begin{tabular}{|c|c|c|c|c|c|c|c|c|c|c|c|c|c|c|c|c|c|c|c|c|c|c|c|c|c|c|c|c|c|c|}
\hline & 1 & 2 & 3 & 4 & 5 & 6 & 7 & 8 & 9 & 10 & 11 & 12 & 13 & 14 & 15 & 16 & 17 & 18 & 19 & 20 & 21 & 22 & 23 & 24 & 25 & 26 & 27 & 28 & 29 & 30 \\
\hline Ch1 & & & & & & P3 & P3 & P3 & P3 & P3 & P3 & P3 & P3 & P3 & P3 & P3 & & P9 & P9 & P9 & & P8 & P8 & P8 & P8 & P8 & P8 & & & \\
\hline Ch2 & & & & & & P7 & P7 & P7 & P7 & P7 & P7 & P7 & P7 & P7 & P7 & P7 & P7 & & & & P10 & P10 & P10 & P1 & P1 & P1 & P1 & P1 & P1 & P1 \\
\hline $\mathrm{Ch} 3$ & & & & & & & & & & & & & & & & & P4 & P4 & P4 & P4 & P4 & P4 & P4 & P4 & P4 & P4 & P4 & P4 & & \\
\hline Ch4 & & & & & & & & & & & & & & & P6 & P6 & P6 & P6 & P6 & P6 & P6 & P6 & $\mathrm{P} 6$ & P6 & P6 & P6 & & & & \\
\hline Ch5 & & & & & & & & & & & & & & P5 & P5 & P5 & & & P2 & P2 & P2 & P2 & P2 & & & & & & & \\
\hline
\end{tabular}

Patients planning

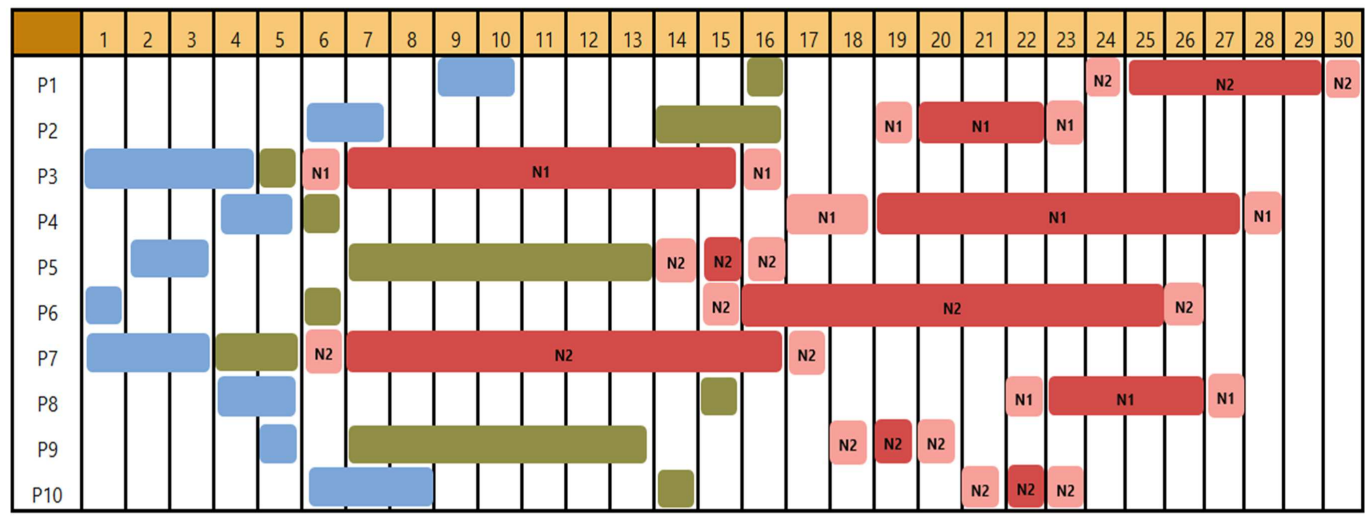

\begin{tabular}{|ll|ll|}
\hline Do & Doctors $0=1 . .3$ & Nj & Nurses $\mathrm{j}=1 . .2$ \\
Chc & Chairs $\mathrm{c}=1 . .5$ & Pk & Patients $\mathrm{k}=1 . .10$ \\
Phi & Pharmacists $\mathrm{i}=1 . .2$ & \\
\hline
\end{tabular}

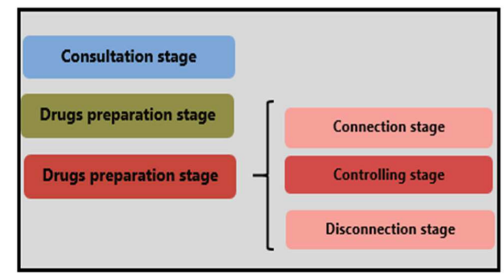

Figure 3. Scheduling solution of Example 1 after applying the constructive heuristic H1. 
Doctors planning

\begin{tabular}{|c|c|c|c|c|c|c|c|c|c|c|c|c|c|c|c|c|c|c|c|c|c|c|c|}
\hline & 1 & 2 & 3 & 4 & 5 & 6 & 7 & 8 & 9 & 10 & 11 & 12 & 13 & 14 & 15 & 16 & 17 & 18 & 19 & 20 & 21 & 22 & 23 \\
\hline D1 & P4 & P4 & P7 & P7 & P7 & P2 & P2 & & & & & & & & & & & & & & & & \\
D2 & P8 & P8 & P5 & P5 & P6 & & & & & & & & & & & & & & & & & & \\
D3 & P3 & P3 & P3 & P3 & P10 & P10 & P10 & P9 & P1 & P1 & & & & & & & & & & & & & \\
\hline
\end{tabular}

Pharmacists planning

\begin{tabular}{|l|l|l|l|l|l|l|l|l|l|l|l|l|l|l|l|l|l|l|l|l|l|l|l|}
\hline & 1 & 2 & 3 & 4 & 5 & 6 & 7 & 8 & 9 & 10 & 11 & 12 & 13 & 14 & 15 & 16 & 17 & 18 & 19 & 20 & 21 & 22 & 23 \\
\hline Ph1 & & & P4 & & P5 & P5 & P5 & P5 & P5 & P5 & P5 & P1 & P2 & P2 & P2 & & & & & & & & \\
Ph2 & & & P8 & & P3 & P6 & P7 & P7 & P10 & P9 & P9 & P9 & P9 & P9 & P9 & P9 & & & & & & & \\
\hline
\end{tabular}

Chairs planning

\begin{tabular}{|l|l|l|l|l|l|l|l|l|l|l|l|l|l|l|l|l|l|l|l|l|l|l|l|}
\hline Ch1 & 1 & 2 & 3 & 4 & 5 & 6 & 7 & 8 & 9 & 10 & 11 & 12 & 13 & 14 & 15 & 16 & 17 & 18 & 19 & 20 & 21 & 22 & 23 \\
Ch2 & & & & P4 & P4 & P4 & P4 & P4 & P4 & P4 & P4 & P4 & P4 & P4 & P4 & & & & P9 & P9 & P9 & & \\
Ch3 & & & & P8 & P8 & P8 & P8 & P8 & P8 & & & P5 & P5 & P5 & & P10 & P10 & P10 & P2 & P2 & P2 & P2 & P2 \\
Ch4 & & & & & & P3 & P3 & P3 & P3 & P3 & P3 & P3 & P3 & P3 & P3 & P3 & P1 & P1 & P1 & P1 & P1 & P1 & P1 \\
Ch5 & & & & & & & P6 & P6 & P6 & P6 & P6 & P6 & P6 & P6 & P6 & P6 & P6 & P6 & & & & & \\
\hline
\end{tabular}

Patients planning

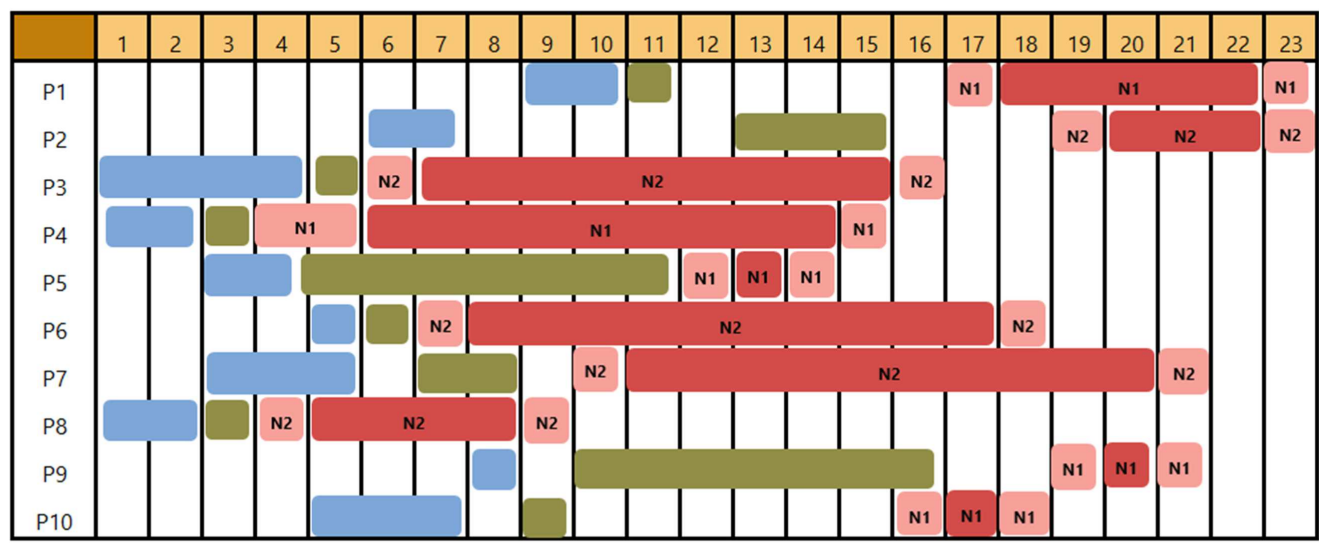

\begin{tabular}{|ll|ll|}
\hline Do & Doctors $0=1 . .3$ & Nj & Nurses $\mathrm{j}=1 . .2$ \\
Chc & Chairs $c=1 . .5$ & Pk & Patients $\mathrm{k}=1 . .10$ \\
Phi & Pharmacists $\mathrm{i}=1 . .2$ & & \\
\hline
\end{tabular}

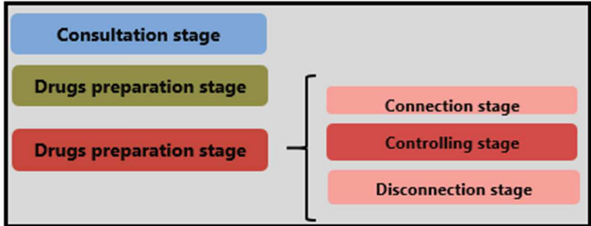

FiguRE 4. Scheduling solution of Example 1 after applying the improvement procedure H2. 
TABle 2. Probability distributions and their parameters for each stage time.

\begin{tabular}{lll}
\hline \hline & Probability distribution & Parameters (min) \\
\hline Consultation & Exponentiel & $\lambda=4.66$ \\
& Expo $(\lambda)$ & \\
Drugs' preparation & Exponentiel & $\lambda=0.67$ \\
& Expo $(\lambda)$ & \\
Injection & Lognormal & $\mu=150$ \\
Setting up & LN2 $(\mu, \sigma)$ & $\sigma=82.10$ \\
& Exponentiel & $\lambda=2.33$ \\
Disconnecting & Expo $(\lambda)$ & \\
& Exponentiel & $\lambda=0.25$ \\
& Expo $(\lambda)$ & \\
\hline
\end{tabular}

For small instances (less than or equal to 15 patients), we compared the mathematical model results with the lower bound (Eq. (5.1)) and the heuristic and metaheuristic. For all instances with greater than or equal to 20 patients, we only compared the heuristic and metaheuristic results with the lower bound.

(H1-LB) and (H1-MIP) determine, respectively, the gap between the lower bound and the solution returned by the heuristic H1 and the gap between the Cplex solution and H1 solution. Similarly, (H2-LB) and (H2-MIP) calculate the gaps between the metaheuristic H2 solution and the lower bound and the Cplex solution.

Tables 3 and 4 display the results for respectively small, medium and large instances.

Table 3, presents instance number (Inst.), the solution given by the lower bound (LB), by Cplex (MIP), by the heuristic (H1) and by the metaheuristic (H2). All completion times are represented in number of slot times. For each subset, we generated ten instances with different referent oncologists and processing times (consultation drug's preparation and injection). For the mathematical model, we detailed the optimal or the best obtained solution, the gap between the obtained solution and the lower bound (Eq. (7.1)), the best Cplex lower bound and the gap between the Cplex lower bound and the calculated lower bound (Eq. (7.2)).

$$
\begin{aligned}
\text { MIP-LB }(\%) & =\text { MIP-LB } / \mathrm{LB} * 100 \\
\text { Cplex-LB }- \text { LB }(\%) & =(\text { Cplex-LB }- \text { LB }) / L B * 100 .
\end{aligned}
$$

For both heuristic and metaheuristic, we detailed the obtained Cmax, the gap with the lower bound (Eqs. (7.3) and (7.5)) and the gap with the mathematical model obtained solution (Eqs. (7.4) and (7.6)).

$$
\begin{aligned}
\mathrm{H} 1-\mathrm{LB}(\%) & =\mathrm{H} 1-\mathrm{LB} / \mathrm{LB} * 100 \\
\mathrm{H} 1-\mathrm{MIP}(\%) & =(\mathrm{H} 1-\mathrm{MIP}) / \mathrm{MIP} * 100 \\
\mathrm{H} 2-\mathrm{LB}(\%) & =(\mathrm{H} 2-\mathrm{LB}) / \mathrm{LB} * 100 \\
\mathrm{H} 2-\mathrm{MIP}(\%) & =(\mathrm{H} 2-\mathrm{MIP}) / \mathrm{MIP} * 100 .
\end{aligned}
$$

The mathematical model gave optimal solutions in $64 \mathrm{~s}$ on average for 5 patients and $3961 \mathrm{~s}$ on average for 10 patients Starting from 15 patients, we were not able to obtain optimal solutions even after $24 \mathrm{~h}$ running time. The program was stopped after $3 \mathrm{~h}$ and both the best solution obtained so far along with the best lower bound were reported. For 5 and 10 patients, the gap between the optimal solution and the lower bound is equal to 096\%, which demonstrates the quality of the lower bound LB. If we include the Cplex best lower bound obtained for 15 patients, the overall gap between the cplex best lower bound and LB is equal to 064\%. We note that for all instances the computational time of both heuristics is negligible (few seconds). If we compare the results of H1 with the optimal solution and the lower bound, the gap with the lower bound is about $13.04 \%$ and with the optimal solution about $10.55 \%$. Regarding the metaheuristic, the results were improved and the gap with the lower bound is only about $5.73 \%$ and with the optimal solution about $3.13 \%$. 
TABLE 3. Results for small instances.

\begin{tabular}{|c|c|c|c|c|c|c|c|c|c|c|c|c|c|}
\hline \multirow[t]{2}{*}{$P$} & \multirow[t]{2}{*}{ Inst. } & \multirow[t]{2}{*}{ LB } & \multicolumn{5}{|c|}{ MIP } & \multicolumn{3}{|c|}{ H1 } & \multicolumn{3}{|c|}{$\mathrm{H} 2$} \\
\hline & & & Cmax & $\begin{array}{l}\text { MIP- } \\
\text { LB } \\
(\%)\end{array}$ & $\begin{array}{l}\text { CPU } \\
(\mathrm{s})\end{array}$ & $\begin{array}{l}\text { Best } \\
\text { Cplex } \\
\text { lower } \\
\text { bound } \\
\end{array}$ & $\begin{array}{l}\text { LB } \\
\text { Cplex- } \\
\text { LB } \\
(\%) \\
\end{array}$ & Cmax & $\begin{array}{l}\text { H1- } \\
\text { LB } \\
(\%)\end{array}$ & $\begin{array}{l}\text { H1- } \\
\text { MIP } \\
(\%)\end{array}$ & Cmax & $\begin{array}{l}\text { H2- } \\
\mathrm{LB} \\
(\%)\end{array}$ & $\begin{array}{l}\mathrm{H} 2- \\
\mathrm{MIP} \\
(\%)\end{array}$ \\
\hline \multirow[t]{10}{*}{$P=5$} & $5-1$ & 41 & 41 & 0.00 & 6 & 41 & 0.00 & 42 & 2.44 & 2.44 & 41 & 0.00 & 0.00 \\
\hline & $5-2$ & 68 & 70 & 2.94 & 237 & 70 & 2.94 & 86 & 26.47 & 22.86 & 74 & 8.82 & 5.71 \\
\hline & $5-3$ & 57 & 57 & 0.00 & 34 & 57 & 0.00 & 57 & 0.00 & 0.00 & 57 & 0.00 & 0.00 \\
\hline & $5-4$ & 67 & 67 & 0.00 & 15 & 67 & 0.00 & 67 & 0.00 & 0.00 & 67 & 0.00 & 0.00 \\
\hline & $5-5$ & 67 & 71 & 5.97 & 84 & 71 & 5.97 & 91 & 35.82 & 28.17 & 87 & 29.85 & 22.54 \\
\hline & $5-6$ & 66 & 66 & 0.00 & 106 & 66 & 0.00 & 71 & 7.58 & 7.58 & 66 & 0.00 & 0.00 \\
\hline & $5-7$ & 54 & 54 & 0.00 & 57 & 54 & 0.00 & 66 & 22.22 & 22.22 & 54 & 0.00 & 0.00 \\
\hline & $5-8$ & 65 & 65 & 0.00 & 32 & 65 & 0.00 & 67 & 3.08 & 3.08 & 65 & 0.00 & 0.00 \\
\hline & $5-9$ & 45 & 45 & 0.00 & 22 & 45 & 0.00 & 66 & 46.67 & 46.67 & 54 & 20.00 & 20.00 \\
\hline & $5-10$ & 59 & 59 & 0.00 & 54 & 59 & 0.00 & 63 & 6.78 & 6.78 & 61 & 3.39 & 3.39 \\
\hline \multirow[t]{10}{*}{$P=10$} & $10-1$ & 44 & 47 & 6.82 & 3155 & 47 & 6.82 & 49 & 11.36 & 4.26 & 49 & 11.36 & 4.26 \\
\hline & $10-2$ & 56 & 56 & 0.00 & 2822 & 56 & 0.00 & 61 & 8.93 & 8.93 & 58 & 3.57 & 3.57 \\
\hline & $10-3$ & 69 & 69 & 0.00 & 5119 & 69 & 0.00 & 82 & 18.84 & 18.84 & 69 & 0.00 & 0.00 \\
\hline & $10-4$ & 57 & 59 & 3.51 & 951 & 59 & 3.51 & 66 & 15.79 & 11.86 & 60 & 5.26 & 1.69 \\
\hline & $10-5$ & 53 & 53 & 0.00 & 3260 & 53 & 0.00 & 54 & 1.89 & 1.89 & 53 & 0.00 & 0.00 \\
\hline & $10-6$ & 66 & 66 & 0.00 & 2366 & 66 & 0.00 & 73 & 10.61 & 10.61 & 66 & 0.00 & 0.00 \\
\hline & $10-7$ & 70 & 70 & 0.00 & 3329 & 70 & 0.00 & 70 & 0.00 & 0.00 & 70 & 0.00 & 0.00 \\
\hline & $10-8$ & 67 & 67 & 0.00 & 5789 & 67 & 0.00 & 68 & 1.49 & 1.49 & 67 & 0.00 & 0.00 \\
\hline & $10-9$ & 67 & 67 & 0.00 & 7934 & 67 & 0.00 & 73 & 8.96 & 8.96 & 68 & 1.49 & 1.49 \\
\hline & $10-10$ & 69 & 69 & 0.00 & 4894 & 69 & 0.00 & 72 & 4.35 & 4.35 & 69 & 0.00 & 0.00 \\
\hline \multirow[t]{10}{*}{$P=15$} & $15-1$ & 69 & $306^{*}$ & & 10800 & 69 & 0.00 & 80 & 15.94 & & 76 & 10.14 & \\
\hline & $15-2$ & 69 & $262^{*}$ & & 10800 & 69 & 0.00 & 100 & 44.93 & & 87 & 26.09 & \\
\hline & $15-3$ & 73 & $249^{*}$ & & 10800 & 73 & 0.00 & 76 & 4.11 & & 76 & 4.11 & \\
\hline & $15-4$ & 69 & $243^{*}$ & & 10800 & 69 & 0.00 & 78 & 13.04 & & 71 & 2.90 & \\
\hline & $15-5$ & 56 & $227^{*}$ & & 10800 & 56 & 0.00 & 68 & 21.43 & & 65 & 16.07 & \\
\hline & $15-6$ & 56 & $228^{*}$ & & 10800 & 56 & 0.00 & 68 & 21.43 & & 62 & 10.71 & \\
\hline & $15-7$ & 72 & $227^{*}$ & & 10800 & 72 & 0.00 & 83 & 15.28 & & 73 & 1.39 & \\
\hline & $15-8$ & 69 & - & & 10800 & 69 & 0.00 & 82 & 18.84 & & 71 & 2.90 & \\
\hline & $15-9$ & 69 & $226^{*}$ & & 10800 & 69 & 0.00 & 69 & 0.00 & & 69 & 0.00 & \\
\hline & $15-10$ & 69 & $227^{*}$ & & 10800 & 69 & 0.00 & 71 & 2.90 & & 71 & 2.90 & \\
\hline Average & & 62.60 & 117.69 & $0.96 \%$ & 4942.20 & 62.97 & $0.64 \%$ & 70.63 & $13.04 \%$ & $10.55 \%$ & 65.87 & $5.37 \%$ & $3.13 \%$ \\
\hline
\end{tabular}

Notes. *Best integer solution obtained by Cplex after $3 \mathrm{~h}$.

In Table 4, the results for medium and large size instances are reported. Only the results of both heuristic and metaheuristic are detailed and compared with the lower bound. The overall gap between H1 solution and the lower bound LB is equal to $32.58 \%$. However, this gap has been improved by the metaheuristic and is equal to $2.51 \%$. The gap is still relatively high and might be justified by some hypotheses. When we increased the number of patients, we did not increase the number of nurses and chairs and maybe it becomes more difficult to find solutions close to the lower bound. We also think that the lower bound is restrictive especially for large size problems and with few resources. Nevertheless, the metaheuristic is capable to determine good solutions very rapidly for a very complex problem. 
TABLE 4. Results for medium and large instances.

\begin{tabular}{|c|c|c|c|c|c|c|}
\hline \multirow[b]{2}{*}{$P$} & \multirow[b]{2}{*}{ Inst. } & \multirow[b]{2}{*}{ LB } & \multicolumn{2}{|r|}{ H1 } & \multicolumn{2}{|r|}{$\mathrm{H} 2$} \\
\hline & & & Cmax & H1-LB (\%) & Cmax & H2-LB (\%) \\
\hline \multirow[t]{10}{*}{$P=20$} & $20-1$ & 68 & 97 & 42.65 & 89 & 30.88 \\
\hline & $20-2$ & 60 & 86 & 43.33 & 80 & 33.33 \\
\hline & $20-3$ & 70 & 96 & 37.14 & 81 & 15.71 \\
\hline & $20-4$ & 75 & 103 & 37.33 & 98 & 30.67 \\
\hline & $20-5$ & 77 & 105 & 36.36 & 101 & 31.17 \\
\hline & $20-6$ & 71 & 92 & 29.58 & 92 & 29.58 \\
\hline & $20-7$ & 70 & 104 & 48.57 & 89 & 27.14 \\
\hline & $20-8$ & 74 & 104 & 40.54 & 100 & 35.14 \\
\hline & $20-9$ & 66 & 94 & 42.42 & 88 & 33.33 \\
\hline & $20-10$ & 62 & 88 & 41.94 & 87 & 40.32 \\
\hline \multirow[t]{10}{*}{$P=25$} & $25-1$ & 76 & 104 & 36.84 & 95 & 25.00 \\
\hline & $25-2$ & 69 & 90 & 30.43 & 90 & 30.43 \\
\hline & $25-3$ & 86 & 118 & 37.21 & 108 & 25.58 \\
\hline & $25-4$ & 65 & 94 & 44.62 & 83 & 27.69 \\
\hline & $25-5$ & 82 & 113 & 37.80 & 110 & 34.15 \\
\hline & $25-6$ & 81 & 114 & 40.74 & 102 & 25.93 \\
\hline & $25-7$ & 70 & 100 & 42.86 & 90 & 28.57 \\
\hline & $25-8$ & 87 & 119 & 36.78 & 104 & 19.54 \\
\hline & $25-9$ & 70 & 98 & 40.00 & 89 & 27.14 \\
\hline & $25-10$ & 84 & 120 & 42.86 & 108 & 28.57 \\
\hline \multirow[t]{10}{*}{$P=30$} & $30-1$ & 114 & 160 & 40.35 & 137 & 20.18 \\
\hline & $30-2$ & 104 & 142 & 36.54 & 136 & 30.77 \\
\hline & $30-3$ & 95 & 124 & 30.53 & 122 & 28.42 \\
\hline & $30-4$ & 93 & 124 & 33.33 & 119 & 27.96 \\
\hline & $30-5$ & 95 & 126 & 32.63 & 121 & 27.37 \\
\hline & $30-6$ & 109 & 141 & 29.36 & 133 & 22.02 \\
\hline & $30-7$ & 85 & 113 & 32.94 & 112 & 31.76 \\
\hline & $30-8$ & 96 & 130 & 35.42 & 120 & 25.00 \\
\hline & $30-9$ & 89 & 129 & 44.94 & 121 & 35.96 \\
\hline & $30-10$ & 107 & 139 & 29.91 & 139 & 29.91 \\
\hline \multirow[t]{10}{*}{$P=35$} & $35-1$ & 104 & 133 & 27.88 & 130 & 25.00 \\
\hline & $35-2$ & 113 & 145 & 28.32 & 139 & 23.01 \\
\hline & $35-3$ & 108 & 145 & 34.26 & 136 & 25.93 \\
\hline & $35-4$ & 103 & 138 & 33.98 & 129 & 25.24 \\
\hline & $35-5$ & 113 & 154 & 36.28 & 142 & 25.66 \\
\hline & $35-6$ & 106 & 148 & 39.62 & 134 & 26.42 \\
\hline & $35-7$ & 110 & 142 & 29.09 & 139 & 26.36 \\
\hline & $35-8$ & 114 & 152 & 33.33 & 139 & 21.93 \\
\hline & $35-9$ & 126 & 174 & 38.10 & 155 & 23.02 \\
\hline & $35-10$ & 132 & 174 & 31.82 & 161 & 21.97 \\
\hline \multirow[t]{2}{*}{$P=40$} & $40-1$ & 120 & 150 & 25.00 & 147 & 22.50 \\
\hline & $40-2$ & 117 & 149 & 27.35 & 147 & 25.64 \\
\hline
\end{tabular}

\section{Conclusion}

In chemotherapy services, it is very important to deliver the right care at the right time that is why scheduling problem for chemotherapy patients is very critical. Our aim is to realize the best health results by minimizing the treatment delays due to limited resources in different stages of the chemotherapy treatment. 
TABLE 4. Continued

\begin{tabular}{|c|c|c|c|c|c|c|}
\hline \multirow[b]{2}{*}{$P$} & \multirow[b]{2}{*}{ Inst. } & \multirow[b]{2}{*}{ LB } & \multicolumn{2}{|r|}{ H1 } & \multicolumn{2}{|r|}{ H2 } \\
\hline & & & Cmax & H1-LB (\%) & Cmax & H2-LB (\%) \\
\hline & $40-3$ & 121 & 163 & 34.71 & 149 & 23.14 \\
\hline & $40-4$ & 112 & 151 & 34.82 & 137 & 22.32 \\
\hline & $40-5$ & 131 & 172 & 31.30 & 160 & 22.14 \\
\hline & $40-6$ & 113 & 145 & 28.32 & 139 & 23.01 \\
\hline & $40-7$ & 142 & 179 & 26.06 & 171 & 20.42 \\
\hline & $40-8$ & 127 & 166 & 30.71 & 160 & 25.98 \\
\hline & 40-9 & 150 & 181 & 20.67 & 181 & 20.67 \\
\hline & $40-10$ & 146 & 193 & 32.19 & 179 & 22.60 \\
\hline \multirow[t]{10}{*}{$P=45$} & $45-1$ & 132 & 171 & 29.55 & 162 & 22.73 \\
\hline & $45-2$ & 167 & 210 & 25.75 & 197 & 17.96 \\
\hline & $45-3$ & 159 & 192 & 20.75 & 192 & 20.75 \\
\hline & $45-4$ & 148 & 188 & 27.03 & 178 & 20.27 \\
\hline & $45-5$ & 140 & 169 & 20.71 & 168 & 20.00 \\
\hline & $45-6$ & 141 & 179 & 26.95 & 175 & 24.11 \\
\hline & $45-7$ & 125 & 156 & 24.80 & 156 & 24.80 \\
\hline & $45-8$ & 135 & 174 & 28.89 & 163 & 20.74 \\
\hline & $45-9$ & 133 & 167 & 25.56 & 158 & 18.80 \\
\hline & $45-10$ & 121 & 154 & 27.27 & 152 & 25.62 \\
\hline \multirow[t]{10}{*}{$P=50$} & $50-1$ & 146 & 179 & 22.60 & 175 & 19.86 \\
\hline & $50-2$ & 150 & 188 & 25.33 & 183 & 22.00 \\
\hline & $50-3$ & 166 & 217 & 30.72 & 203 & 22.29 \\
\hline & $50-4$ & 169 & 210 & 24.26 & 202 & 19.53 \\
\hline & $50-5$ & 153 & 199 & 30.07 & 186 & 21.57 \\
\hline & $50-6$ & 149 & 188 & 26.17 & 176 & 18.12 \\
\hline & $50-7$ & 155 & 196 & 26.45 & 191 & 23.23 \\
\hline & $50-8$ & 157 & 200 & 27.39 & 187 & 19.11 \\
\hline & 50-9 & 154 & 193 & 25.32 & 188 & 22.08 \\
\hline & $50-10$ & 154 & 193 & 25.32 & 187 & 21.43 \\
\hline Average & & 111.60 & 146.34 & $32.58 \%$ & 138.53 & $25.10 \%$ \\
\hline
\end{tabular}

To the best of our knowledge, there is no study that proposes optimization methods to schedule patients considering all stages of chemotherapy treatment with human and material resources management.

We proposed an exact resolution for scheduling patients for multiple stages in an outpatient chemotherapy department. The proposed scheduling method improves the patient flow by reducing the makespan, which is a critical factor. In current practice, appointments specified only the treatment day, which increases the total waiting time and the completion time. A shorter time between the arrival time and appointment time can reduce the waiting times for patients so we propose to determine the appointment time for each patient using a MIP model. The objective is to minimize the completion time of the daily activity. The model is formulated then tested with Cplex. It gave optimal solutions for less than or equal to than 10 patients. In this model, we integrate all stages of chemotherapy treatment based on instances from real world. In our case (private context), the development of exact methods can be sufficient especially at the end of the week when the patient volume is not high. For peak days (high volume of patients), we proposed a metaheuristic, that can reach satisfying solutions in a very short time. To assess the quality of the metaheuristic, we proposed a good-quality lower bound. 
We led then an experimental study and show that our metaheuristic had a good performance for small instances with about $5.37 \%$ gap to the lower bound and $3.13 \%$ gap to the optimal solution. The gap to the lower bound increases to about $25 \%$ for medium and large instances.

The next step of this work is to improve the metaheuristic to get lower gaps with the lower bound for large size problems.

Other objective functions can also be considered such as reducing patients' waiting times, maximizing the number of admitted patients, and maximizing profits.

In practice, chemotherapy process times are highly variable and uncertain. Thus, accounting for this random behavior in patients' scheduling is crucial. For future research, we aim then to study chemotherapy scheduling problem under uncertainty in the processing times of each stage. Stochastic and scenario based approaches can be considered.

Acknowledgements. The authors are grateful to the reviewers for their careful reading of the paper and their valuable feedback.

\section{REFERENCES}

[1] Z. Ahmed, T.Y. ElMekkawy and S. Bates, Developing an efficient scheduling template of a chemotherapy treatment unit: a case study. Australas. Med. J. 4 (2011) 575-588.

[2] M. Benzaid, N. Lahrichi and L.M. Rousseau, Chemotherapy appointment scheduling and daily outpatient-nurse assignment. Health Care Manag. Sci. 23 (2020) 34-50.

[3] A. Condotta and N.V. Shakhlevich, Scheduling patient appointments via multilevel template: a case study in chemotherapy. Oper. Res. Health Care 3 (2014) 129-144.

[4] J. Connell, Contemporary medical tourism: conceptualisation culture and commodification. Tourism Manage. 34 (2013) 1-13.

[5] R. Donahue, D. Russell, C. de Riese, C. Smith, W.T.W de Riese and A. Medway, Patients willing to wait: arrival time wait time and patient satisfaction in an ambulatory urology clinic. Urology Pract. 4 (2017) 1-6.

[6] J. Framinan, J. Gupta and R. Leisten, A review and classification of heuristics for permutation flow-shop scheduling with makespan objective. J. Oper. Res. Soc. 55 (2004) 1243-1255.

[7] T. Garaix, S. Rostami and X. Xie, Daily outpatient chemotherapy appointment scheduling with random deferrals. Flexible Serv. Manuf. J. 38 (2020) 1-25.

[8] M.R. Garey, D.S. Johnson and D.R. Sethi, The complexity of flowshop and job shop scheduling. Math. Oper. Res. 1 (1976) $117-129$

[9] F. Glover, Tabu search - Part I. ORSA J. Comput. 1 (1989) 129-153.

[10] R.L. Graham, E.L. Lawler, J.K. Lenstra and A. Kan, H. G. R. . Optimization and approximation in deterministic sequencing and scheduling: a survey. Ann. Discrete Math. 5 (1979) 287-326.

[11] C. Granja, B. Almada-Lobo, F. Janela, J. Seabra and A. Mendes, An optimization based on simulation approach to the patient admission scheduling problem using a linear programing algorithm. J. Biomed. Inf. 52 (2014) 427-437.

[12] J.N.D. Gupta, Two-stage hybrid flow-shop scheduling problem. J. Oper. Res. Soc. 389 (1988) 359-364.

[13] S. Hahn-Goldberg, M. Carter, J.C. Beck, M. Trudeau, P. Sousa and K. Beattie, Dynamic optimization of chemotherapy outpatient scheduling with uncertainty. Health Care Manag. Sci. 17 (2014) 379-392.

[14] A.F. Hesaraki, N.P. Dellaert and T. de Kok, Generating outpatient chemotherapy appointment templates with balanced flowtime and makespan. Eur. J. Oper. Res. 275 (2019) 304-318.

[15] L. Hopkins, R. Labonté, V. Runnels and C. Packer, Medical tourism today: What is the state of existing knowledge. J. Publ. Health Policy 31 (2010) 185-198.

[16] J.A. Hoogeveen, J.K. Lenstra and B. Veltman, Preemptive scheduling in a two-stage multiprocessor flow shop is NP-hard. Eur. J. Oper. Res. 89 (1996) 172-175.

[17] Y.-L. Huang, A.H. Bryce, T. Culbertson, S.L. Connor, S.A. Looker, K.M. Altman, J.G. Collins, W. Stellner, R.R. McWilliams, A. Moreno-Aspitia, S. Ailawadhi and R.A. Mesa, Alternative outpatient chemotherapy scheduling method to improve patient service quality and nurse satisfaction. J. Oncol. Pract. 14 (2018) 82-91.

[18] Y. Huang, S. Bach and S. Looker, Chemotherapy scheduling template development using an optimization approach. Int. J. Health Care Qual. Assur. 32 (2019) 59-70.

[19] R. Kunwar Ramesh, Medical tourism and hospitality in hospital the gaze. J. Tourism Hospitality 10 (2019) 67-123.

[20] M. Laguna, J.W. Barnes and F.W. Glover, Tabu search methods for a single machine scheduling problem. J. Intell. Manuf., 2 (1991) 63-73.

[21] F. Li, D. Gupta and S. Potthoff, Improving operating room schedules. Health Care Manag. Sci. 19 (2016) $261-278$.

[22] B. Liang and A. Turkcan, Acuity-based nurse assignment and patient scheduling in oncology clinics. Health Care Manag. Sci. 19 (2016) 207-226. 
[23] W.J. Mackillop, Killing time: the consequences of delays in radiotherapy. Radiother. Oncol. 84 (2007) 1-4.

[24] K.D. Miller, R.L. Siegel, C.C. Lin, A.B. Mariotto, J.L. Kramer, J.H. Rowland, K.D. Stein, R. Alteri and A. Jemal, Cancer treatment and survivorship statistics CA: a cancer. J. Clin. 69 (2016) 363-385.

[25] L. Minh-Duc, H. Minh, N. Nhat, B. Chantal, G. Viviane and D. Tien Ba, Heuristics to solve appointment scheduling in chemotherapy. In: 2015 IEEE RIVF International Conference, Can Tho, Vietnam (25-28 Jan 2015).

[26] J.J. Mogaka, T.P. Mashamba-Thompson, J.M. Tsoka-Gwegweni and L.M. Mupara, Tourism and leisure effects of medical tourism on health systems in Africa. Afr. J. Hospitality 6 (2017) 1-25.

[27] C. Oguz and M.F. Ercan, A genetic algorithm for hybrid flow-shop scheduling with multiprocessor tasks. J. Scheduling 8 (2005) 323-351.

[28] J. Ragaz, I.A. Olivotto, J.J. Spinelli, N. Phillips, S.M. Jackson, K.S. Wilson, M.A. Knowling, C.M.L. Coppin, L. Weir, K. Gelmon, N. Le, R. Durand, A.J. Coldman and M. Manji, Locoregional radiation therapy in patients with high-risk breast cancer receiving adjuvant chemotherapy: 20-year results of the British Columbia randomized trial. J. Nat. Cancer Inst. 97 (2005) 116-126.

[29] A. Ranerup, H. Zinner Henriksen and J. Hedman, An analysis of business models in Public Service Platforms. Government Inf. Q. 33 (2016) 6-14.

[30] G. Ridic, S. Gleason and O. Ridic, Comparisons of health care systems in the United States, Germany and Canada. Mater. Soc. Med. 24 (2012) 112-120.

[31] R. Ruiz and J.A. Vázquez-Rodríguez, The hybrid flow shop scheduling problem. Eur. J. Oper. Res. 205 (2010) 1-18.

[32] A. Sadki, X. Xie and F. Chauvin, Planning oncologists of ambulatory care units. Decis. Support Syst. 55 (2013) 640-649.

[33] R.B. Saltman, Melting public-private boundaries in European health systems. Eur. J. Publ. Health 13 (2003) $24-29$.

[34] P. Santibáñez, R. Aristizabal, M.L. Puterman, V.S. Chow, W. Huang, C. Kollmannsberger, T. Nordin, N. Runzer and S. Tyldesley, Operations research methods improve chemotherapy patient appointment scheduling. Jt. Comm. J. Qual. Patient Saf. 38 (2012) 541-553.

[35] S. Shashaani, Chemotherapy patient scheduling and uncertainty. Master thesis, Purdue University (2011).

[36] S. Shahnawaz, Optimization of the patients appointments in chemotherapy treatment unit: heuristic and metaheuristic approaches. Master thesis, University of Manitoba (2012).

[37] C.S. Sung and H.A. Kim, A two-stage multiple-machine assembly scheduling problem for minimizing sum of completion times. Int. J. Prod. Econ. 113 (2008) 1038-1048.

[38] T. Tanaka, Infusion chair scheduling algorithms based on bin-packing heuristics. Master thesis, Binghamton University (2013).

[39] A. Turkcan, B. Zeng and M. Lawley, Chemotherapy Operations Planning and Scheduling. IIE Trans. Healthc. Syst. Eng. 2 (2012) 31-49.

[40] H.M. Wang, F.D. Chou, F.C. Wu and M.Y. Ku, Hybrid flow shop scheduling problems with multiprocessor tasks. Appl. Mech. Mater. 110 (2011) 3914-3921.

[41] J.C. Woodall, T. Gosselin, A. Boswell, M. Murr and B.T. Denton, Improving patient access to chemotherapy treatment at Duke Cancer Institute. Interfaces 43 (2013) 449-461.

[42] K.-C. Ying and S.-W. Lin, Scheduling multistage hybrid flowshops with multiprocessor tasks by an effective heuristic. Int. J. Prod. Res. 47 (2009) 3525-3538.

[43] M. Yokouchi, S. Aoki, H. Sang, R. Zhao and S. Takakuwa, Operations analysis and appointment scheduling for an outpatient chemotherapy department. In: Proceedings of the 2012 Winter Simulation Conference, Berlin, Germany (February 2013) 907918. 